\title{
Genetic variation and phylogenetic analysis of Porcine circovirus type 2 in China from 2016 to 2018
}

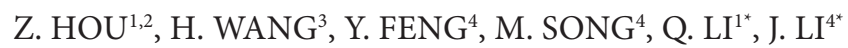

\begin{abstract}
${ }^{1}$ College of Animal Science and Technology, Northwest A\&F University, Yangling, 712100, P. R. China; ${ }^{2}$ College of Marine Science and Engineering, Qingdao Agricultural University, Qingdao, 266109, P. R. China; ${ }^{3}$ Qingdao Vland Biotech Group Co. Ltd., Qingdao, 266061, P. R. China; ${ }^{4}$ College of Veterinary Medicine, Qingdao Agricultural University, Qingdao, 266109, P. R. China
\end{abstract}

Received February 2, 2019; accepted April 3, 2019

\begin{abstract}
Summary. - Infection with Porcine circovirus type 2 (PCV2), the essential cause of porcine circovirus (PCV) associated diseases, is a growing problem in swine industry around the world. High nucleotide alteration leads to the constant evolution of PCV2 and outbreak of disease caused by PCV2. In this study, 48 PCV2 strains were isolated in China between 2016 and 2018 and the genetic diversity of these PCV2 isolates was determined. Results showed that these PCV2 isolates were classified into genotypes PCV2a (4 of 48), PCV2b (13 of 48) and PCV2d (31 of 48). Among them, 54.5\% isolated in 2016, 65.2\% isolated in 2017 and $71.4 \%$ isolated in 2018 belonged to PCV2d. It indicated that the prevalence of PCV2d genotype increased. All strains shared $93.4 \%-100 \%$ nucleotide sequence identity for the whole genome. Results of the analysis using RDP 4.0 molecular recombination software showed there were no recombinant events among the 48 PCV2 isolates in this study. Further analysis indicated the presence of higher amino acid residues diversity in important epitopes $\left({ }^{43} \mathrm{D} / \mathrm{G},{ }^{115} \mathrm{D} / \mathrm{G},{ }^{134} \mathrm{~N} / \mathrm{T},{ }^{165} \mathrm{P} / \mathrm{T},{ }^{169} \mathrm{G} / \mathrm{R} / \mathrm{S}\right.$, and $\left.{ }^{210} \mathrm{E} / \mathrm{G} / \mathrm{D}\right)$ in the predominant genotype PCV2d. Animal tests showed the viral titer of the PCV2d strain LN-3 in sera was higher than that of PCV2b strain HeB-1 at 14 and 21 days post-challenge, however, the differences were not statistically significant. There were also no obvious differences between PCV2d strain LN-3 and PCV2b strain HeB-1 in the amount of PCV2 antigen in lymphoid tissues. On the account of the increasing prevalence of PCV2d genotype, it is necessary to find the cause of PCV2 genotype change, to evaluate the effect of existing commercial vaccines and to develop new vaccines based on PCV2d genotype, if necessary.
\end{abstract}

Keywords: epitope; PCV2d; phylogeny; Porcine circovirus type 2; recombination; genetic diversity

\section{Introduction}

Infection by Porcine circovirus type 2 (PCV2) in pigs results in a variety of diseases, such as post-weaning multisystemic wasting syndrome (PMWS) (Afolabi et al., 2017), porcine dermatitis and nephropathy syndrome (PDNS)

*Corresponding authors. E-mail: liqingwangysu@aliyun.com (Qingwang Li); Jwli@qau.edu.cn (Junwei Li); phone: +8613465445572.

Abbreviations: $\mathrm{ORF}(\mathrm{s})=$ open reading frame(s); PCV2 = Porcine circovirus type 2; PCVADs = PCV-associated diseases; PMWS $=$ post-weaning multi-systemic wasting syndrome
(Kwon et al., 2017), porcine respiratory disease complex (PRDC), and other clinical disease manifestations that have generally been classified as Porcine circovirus-associated diseases (PCVADs) (Opriessnig et al., 2007). Since late 1990s, PCV2 has developed into a major pig pathogen and caused significant economic losses to the swine industry worldwide (Tischer et al., 1982).

PCV2 belongs to the family Circoviridae, the genus Cirovirus and is a small, non-enveloped virus containing a covalently closed, circular, single-stranded DNA genome (Trible et al., 2011). The genome of PCV2 contains 1766-1768 nt with at least five potential open reading frames (ORFs) (Trible et al., 2011). The largest (ORF1) encodes the replicase proteins (Rep), Rep and Rep'. Rep is translated from the entire ORF1, 
while Rep' is encoded by an internally spliced RNA of ORF1 (Cheung, 2012). ORF2 encodes the structural protein of the capsid, which is the only component of the viral capsid and the major target of the host immune response (Nawagitgul et al., 2000; Franzo et al., 2016). ORF3 is not essential for PCV2 replication but is involved in PCV2-induced apoptosis by activating both the caspase- 8 and caspase- 3 pathways. ORF4 plays a role in suppressing the caspase activity and regulating CD4 and CD8 T lymphocytes during PCV2 infection. The ORF5 gene has a length of $180 \mathrm{bp}$ and overlaps completely with ORF1 when read in the same direction. Its protein is degraded via the proteasome, inhibits porcine alveolar macrophage (PAM) growth and prolongs the S-phase of the cell cycle (Lv et al., 2015).

PCV2 is continuously evolving through a series of point mutations and gene recombinations in the genome. The rate of the nucleotide alteration for PCV2 has been calculated to be about $1.2 \times 10^{-3}$ substitutions per site and year. This is relatively high substitution rate for a single-stranded DNA virus, which enables PCV2 to have evolutionary dynamics closer to that of single-stranded RNA viruses than to that of double-stranded DNA viruses (Firth et al., 2009). Based on the proportion of different nucleotide sites in ORF2 from viruses from various countries, PCV2 strains could be subdivided into three main genotypes (PCV2a, PCV2b, and PCV2c) and two new genotypes (PCV2d and PCV2e) (Olvera et al., 2007; Davies et al., 2016; Zhan et al., 2016).

For prevention of PCV2 infection, vaccination has been proven to be the most effective way to mitigate losses and to decrease PCV2 prevalence in the field. Although PCV2 vaccines can reduce clinical signs of PCVAD, the emergence of new strains (some with increased virulence) fuels the debate that PCV2 vaccines need a regular update including new strains. Information about the genetic variation of PCV2 in pig herds is critical for vaccine development and PCVAD prevention. The published data showed that PCV2a vaccination can provide a significant protection to animals challenged with PCV2b and PCV2d (Ramos et al., 2013; Segales et al., 2013); furthermore, genotype 2b-based PCV2 vaccine is more effective than vaccine based on $P C V 2 a$ for protection against PCV $2 \mathrm{~b}$ or combined PCV2a/2b viremia in pigs (Opriessnig et al., 2013). Nevertheless, PCV2 is still circulating in vaccinated farms. Due to the high mutation rate of the virus, recent findings indicate concerns that PCV2 strains may be capable to escape vaccination (Rose et al., 2016). Phylogenetic analysis is a powerful tool and is currently widely used to investigate antigens and capsid structure variations in viruses. The main purpose of this study was the investigation of the genetic variation and phylogenetic characteristics of PCV2 in China from 2016 to 2018 , and to improve the research for diagnosis, vaccine design, and PCV2 pathogenesis.

\section{Materials and Methods}

Research involving animal subjects. All animal samples were collected in accordance with the standards of laboratory animal care (2016) in China and other related regulations in Animal Welfare Act (Approval No. 20180025). The animal study proposal was approved by the Institutional Animal Care and Use Committee (IACUC) of the Shandong province. All animal care and experiments were carried out in accordance with the Regulations for the Administration of Affairs Concerning Experimental Animals approved by the State Council of People's Republic of China.

Sample collection. A total of 217 clinical samples consisting of lung, mesenteric lymph node, and tonsil tissue were collected during 2016-2018 from 65 farms in China, including Shandong, Hunan, Hubei, Henan, Hebei, Tianjin, Anhui, Jiangsu, Zhejiang, Jilin, Guangdong, Yunnan, Liaoning Heilongjiang, Inner Mongolia, Shanxi, Gansu, Jiangxi, Sichuan, and Shanghai provinces, for the detection of PCV2 infection. Fresh samples were randomly collected from diseased pigs of the sampled farms, were processed and stored at $-80^{\circ} \mathrm{C}$ until further use.

PCV2 genomic DNA amplification and sequencing. Viral genomic DNA was extracted from fresh or frozen tissue samples using a DNA viral Genome Extraction Kit (Solarbio, Beijing, China) according to the manufacturer's instruction. The PCV2 genomic DNA was PCR-amplified using the primer pairs described in Guo et al. (Guo et al., 2010). The resultant PCR products were purified using a Gel Extraction Kit (Solarbio, Beijing, China) according to the manufacturer's protocol, and then ligated to the pMD18-T Vector (Takara, Dalian, China) at $16^{\circ} \mathrm{C}$ for 2 hours. The ligation products were transformed into $\mathrm{DH} 5 a$ chemically competent cells and positive plasmid-containing inserts were identified via PCR. Positive bacterial suspensions were sent to a commercial facility (Sangon Biotech, Shanghai, China) for sequencing.

Genetic variation and phylogenetic analyses of PCV2 sequences. The sequences of 48 PCV2 isolates from 2016 to 2018, 25 other sequences obtained from GenBank, and the sequences of PCV2 ORF2 were aligned and analyzed using the Clustal W method of the Megalign program (DNAStar software). A neighbor-joining tree was constructed using MEGA7.0 with 1000 bootstrap replicates and the Kimura 2-parameter nucleotide substitution model (Kumar et al., 2016).

Recombination between PCV2 sequences. To detect possible recombination events within the 48 PCV2 genomes obtained from 2016 to 2018 and 25 other sequences obtained from GenBank, seven methods implemented in the Recombination Detection Program (RDP) 4 were utilized including RDP, GeneConv, Bootscan, Maxchi, Chimaera, SiSscan, and 3Seq (Martin et al., 2015). The following settings were employed in these analyses: window size $=20$, highest multiple comparison-corrected $p$-value $=0.01$, Bonferroni correction, finding consensus daughter sequences, and polishing breakpoints. Only putative recombination events detected by more than one method were considered. Pathogenicity study of the PCV2b strain HeB-1 and PCV2d strain LN-3. To determine the 
pathogenic differences between PCV2b strain HeB-1 and PCV2d strain LN-3, fifteen four-week-old piglets were randomly divided into three groups and maintained in individual rooms. All piglets were negative for antigen and antibody of Porcine circovirus type 2 (PCV2) before the experiment. Each piglet in the PCV2 strain group (HeB-1 or LN-3) was challenged intranasally with $2.0 \mathrm{ml}$ of the PCV2b strain HeB-1 and PCV2d strain LN-3 at a titer of 105.5 $\mathrm{TCID}_{50} / \mathrm{ml}$. Each animal in the control group was given the same dosage of PK-15 cell culture medium. Piglets were monitored for 21 days after challenge and then were euthanized. The viremia of PCV2-challenged animals was detected at $0,7,14$, and 21 day post-challenge. Viral DNA in serum samples was extracted using QIAamp DNA Mini Kit (Qiagen, Hilden, Germany) according to the manufacturer's instructions and subjected to a SYBR green I PCR for the detection of PCV2 (Li et al., 2015). PCV2 antigen in Lymphoid nodes was detected by PCV2 immunohistochemistry (IHC). Briefly, thin paraffin sections $(5 \mu \mathrm{m})$ collected from each lymph node were dewaxed in xylene and rehydrated in ethanol (100\%, 95\%, 90\%, 80\% and 70\%) for $10 \mathrm{~min}$. After treatment with $3 \% \mathrm{H}_{2} \mathrm{O}_{2}$ for $10 \mathrm{~min}$ and a block, all slides were incubated in primary antibody (porcine polyclonal anti-PCV2 antibody) for $2 \mathrm{~h}$ and in secondary antibody (HRP-labeled goat anti-porcine IgG) for $1 \mathrm{~h}$ at room temperature. The sections were stained with $\mathrm{DAB}$ solution and then counterstained with hematoxylin.

\section{Results}

\section{PCV2 detection}

Of 217 samples collected in China from 2016 to 2018, 48 samples tested positive for PCV2. Data of PCV2 isolates characterized in this work were ordered according to geographic origin, age, isolate tissue, clinical history, and the year of sample collection; these data are listed in Table 1.

Genetic variation and phylogenetic analysis of PCV2 sequences

Phylogenetic analysis based on the PCV2 complete genome (Fig. 1) and ORF2 (Fig. 2) indicated that $8.3 \%$ (4/48), $27.1 \%(13 / 48)$, and $64.6 \%(31 / 48)$ of the 48 PCV2 isolates belonged to genotype PCV2a, PCV2b, and PCV2d,

\section{Fig. 1}

Phylogenetic tree based on the complete genome of PCV2 isolates from China

The tree was constructed using 73 partial genomes of PCV2 (1041 nt), which comprised 48 strains from this study and 25 other sequences obtained from GenBank. PCV1 sequence served as an out-group. The construction was obtained using a Neighbor Joining algorithm. The percentage of replicate trees in which the associated taxa clustered together in the bootstrap test (1000 replicates) are shown next to the branches.

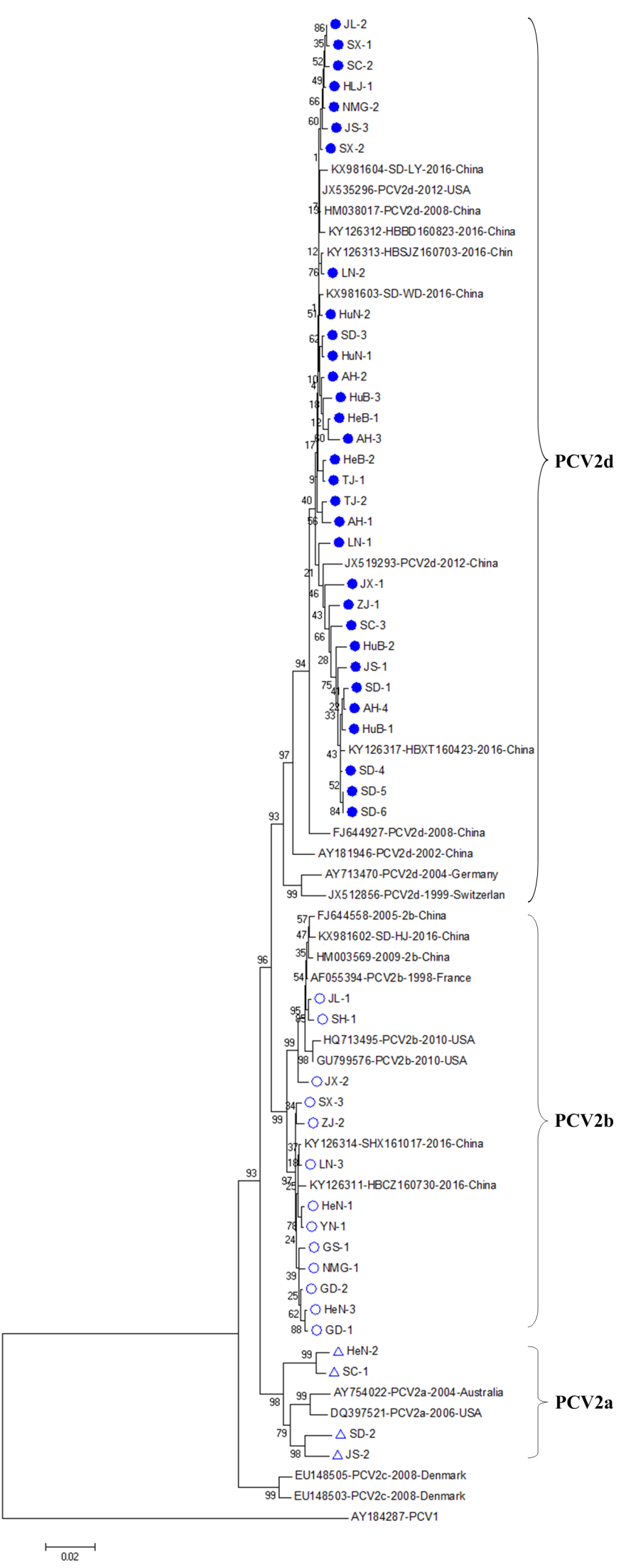




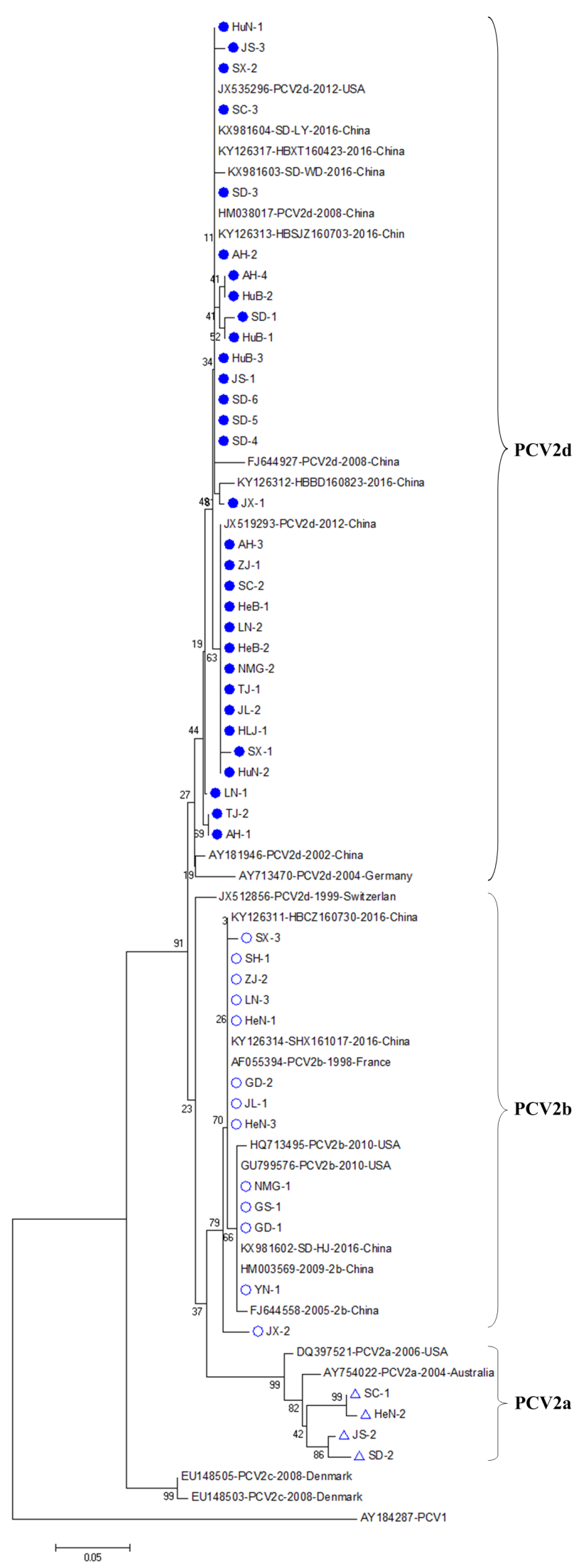

respectively. In addition, $54.5 \%$ isolates belonged to PCV2d in 2016 , $65.2 \%$ in 2017 , and $71.4 \%$ in 2018 . The PCV $2 d$ isolates were the predominant genotype that circulated in China from 2016 to 2018 in our investigation. The nucleotide homology levels of PCV2a, PCV2b, and PCV2d were 94.6-97.9\%, 97.9-99.8\%, and 97.7-100\%, respectively. All strains shared 93.4-100\% nucleotide sequence identity for the whole genome (Table 2). These results indicate that the genome of PCV2a contained the highest variability while PCV2d exhibited minimum variability in terms of nucleotide homology among Chinese PCV2 genotypes. Genetic divergence of the genome of these 48 isolates from China PCV2 genotypes were calculated as 94.5-99.8\% (PCV2a and PCV2b), 93.4-100\% (PCV2a and PCV2d), and 95.4-100\% (PCV2b and PCV2d).

\section{Recombination analysis}

Recombination is a strong contributing force to the evolution of viruses and their genetic diversity. Based on RDP 4.0 molecular recombination software, six potential recombination events were identified amongst genomes of 48 PCV2 isolates and 25 other sequences obtained from GenBank (Table 3). One of these recombination events involved exchanges between PCV2b and PCV2d sequences, while another recombination event involved exchanges between PCV2c and PCV2d, both recombination events involved exchanges between PCV2d sequences. However, regardless of whether exchange happened between PCV2d or PCV2b and PCV2d sequences, the four recombinants were of PCV2d genotype. Interestingly, three recombinant strains (KY126314-SHX16, JX512856-PCV2d, and AY754022PCV2a) were derived from the potential parental strain JX519293-PCV2d. In addition, no other recombinants were found in the 48 isolated strains described in this study.

\section{Analysis of Cap amino acid sequences}

To investigate amino acid variations of capsid among all 48 PCV2 isolates in China from 2016 to 2018, the deduced Cap amino acid sequences were aligned and 53 variable amino acid residues regions of Cap were detected (Table 4). Cap had four antibody recognition domains, including po-

Fig. 2

Phylogenetic tree based on the ORF2 sequence of PCV2 isolates from China

The tree was constructed using 73 sequences of PCV2 ORF2 (1041 nt), which comprised 48 strains from this study and 25 other sequences obtained from GenBank. PCV1 sequence served as an out-group. The construction was obtained using a Neighbor Joining algorithm. The percentage of replicate trees in which the associated taxa clustered together in the bootstrap test (1000 replicates) are shown next to the branches. 
Table 1. Identification of 48 PCV2 isolates from different geographic regions in China - materials with clinical history

\begin{tabular}{|c|c|c|c|c|c|c|c|}
\hline No. & Isolates name & Isolate region & Age (weeks) & Clinical history & Isolate tissue & Isolate time & GenBank No. \\
\hline 1 & SD-1 & Shandong & $6-7$ & PMWS & Spleen, lymphnode, lung & 2016 & MH890676 \\
\hline 2 & SD-2 & Shandong & $6-7$ & PMWS & Spleen, lymphnode, lung & 2016 & MH920544 \\
\hline 3 & SD-3 & Shandong & $3-4$ & Dead pig & Lymph node & 2017 & MH931449 \\
\hline 4 & SD-4 & Shandong & $3-4$ & Dead pig & Lymph node & 2017 & MH920545 \\
\hline 5 & SD-5 & Shandong & $3-4$ & Dead pig & Lymph node & 2017 & MH920546 \\
\hline 6 & SD-6 & Shandong & 5 & PMWS & Spleen, lymph node, lung & 2018 & MH920547 \\
\hline 7 & $\mathrm{HeN}-1$ & Henan & $3-5$ & PMWS & Spleen, lymphnode, lung & 2016 & MH920548 \\
\hline 8 & $\mathrm{HeN}-2$ & Henan & 7 & Respiratory signs & Serum & 2017 & MH920589 \\
\hline 9 & HeN-3 & Henan & 7 & Respiratory signs & Serum & 2017 & MH920549 \\
\hline 10 & HeB-1 & Hebei & Unknown & Dead pig & Lymph, node & 2017 & MH920550 \\
\hline 11 & $\mathrm{HeB}-2$ & Hebei & Unknown & Dead pig & Lymph node & 2017 & MH920551 \\
\hline 12 & TJ-1 & Tianjin & $6-7$ & PMWS & Spleen, lymph node, lung & 2017 & MH920552 \\
\hline 13 & $\mathrm{TJ}-2$ & Tianjin & Unknown & PMWS & Spleen, lymphnode, lung & 2018 & MH920553 \\
\hline 14 & AH-1 & Anhui & $5-6$ & PMWS & Spleen, lymphnode, lung & 2016 & MH920554 \\
\hline 15 & AH-2 & Anhui & $5-6$ & Respiratory signs & Serum & 2017 & MH920555 \\
\hline 16 & AH-3 & Anhui & $5-6$ & PMWS & Spleen, lymphnode, lung & 2017 & MH920556 \\
\hline 17 & $\mathrm{AH}-4$ & Anhui & Unknown & PMWS & Spleen, lymph node, lung & 2018 & MH920557 \\
\hline 18 & HuB-1 & Hubei & $5-6$ & PMWS & Spleen, lymphnode, lung & 2017 & MH920558 \\
\hline 19 & HuB-2 & Hubei & $5-6$ & PMWS & Spleen, lymphnode, lung & 2017 & MH920559 \\
\hline 20 & HuB-3 & Hubei & $5-6$ & Dead pig & Lymph node & 2018 & MH920560 \\
\hline 21 & HuN-1 & Hunan & Unknown & PMWS & Spleen, lymphnode, lung & 2017 & MH920561 \\
\hline 22 & HuN-2 & Hunan & Unknown & PMWS & Spleen, lymph node, lung & 2017 & MH920562 \\
\hline 23 & GD-1 & Guangdong & 3 & Dead pig & Lymph node & 2017 & MH920563 \\
\hline 24 & GD-2 & Guangdong & 3 & Dead pig & Lymph node & 2018 & MH920564 \\
\hline 25 & $\mathrm{YN}-1$ & Yunnan & Unknown & Dead pig & Lymph node & 2016 & MH920565 \\
\hline 26 & $\mathrm{LN}-1$ & Liaoning & Unknown & Dead pig & Lymph node & 2016 & MH920566 \\
\hline 27 & $\mathrm{LN}-2$ & Liaoning & Unknown & Swine fever & Serum & 2017 & MH920567 \\
\hline 28 & LN-3 & Liaoning & Unknown & Swine fever & Serum & 2017 & MH920568 \\
\hline 29 & JL-1 & Jilin & 7 & PMWS & Spleen, lymph node, lung & 2018 & MH920569 \\
\hline 30 & JL-2 & Jilin & 7 & PMWS & Spleen, lymph node, lung & 2018 & MH920570 \\
\hline 31 & HLJ-1 & Heilongjiang & 5 & PMWS & Spleen, lymph node, lung & 2018 & MH920571 \\
\hline 32 & NMG-1 & Inner Mongolia & 3 & Dead pig & Lymph node & 2016 & MH920572 \\
\hline 33 & NMG-2 & Inner Mongolia & 3 & Dead pig & Lymph node & 2016 & MH920573 \\
\hline 34 & SX-1 & Shanxi & 5 & PMWS & Spleen, lymphnode, lung & 2017 & MH920574 \\
\hline 35 & SX-2 & Shanxi & $5-6$ & Dead pig & Lymph node & 2018 & MH920575 \\
\hline 36 & SX-3 & Shanxi & $5-6$ & Dead pig & Lymph node & 2018 & MH920576 \\
\hline 37 & GS-1 & Gansu & Unknown & PMWS & Spleen, lymph node, lung & 2017 & MH920577 \\
\hline 38 & JS-1 & Jiangsu & Unknown & Dead pig & Lymph node & 2016 & MH920578 \\
\hline 39 & JS-2 & Jiangsu & $5-6$ & Dead pig & Lymph node & 2017 & MH920579 \\
\hline 40 & JS-3 & Jiangsu & $5-6$ & Dead pig & Lymph node & 2018 & MH920580 \\
\hline 41 & JX-1 & Jiangxi & $3-4$ & PMWS & Spleen, lymph node, lung & 2018 & MH920581 \\
\hline 42 & $\mathrm{JX}-2$ & Jiangxi & $3-4$ & PMWS & Spleen, lymph node, lung & 2018 & MH920582 \\
\hline 43 & ZJ-1 & Zhejiang & Unknown & PMWS & Spleen, lymph node, lung & 2016 & MH920583 \\
\hline 44 & ZJ-2 & Zhejiang & Unknown & PMWS & Spleen, lymph node, lung & 2016 & MH920584 \\
\hline 45 & SC-1 & Sichuan & 4 & Dead pig & Lymph node & 2017 & MH920585 \\
\hline 46 & $\mathrm{SC}-2$ & Sichuan & 4 & Dead pig & Lymph node & 2017 & MH920586 \\
\hline 47 & SC-3 & Sichuan & Unknown & PMWS & Spleen, lymph node, lung & 2018 & MH920587 \\
\hline 48 & SH-1 & Shanghai & $5-6$ & Dead pig & Lymph node & 2017 & MH920588 \\
\hline
\end{tabular}


Table 2. Nucleotide homology levels (percent) of 48 PCV2 isolates and 25 other sequences obtained from GenBank

\begin{tabular}{lccccccc}
\hline & Whole genome & PCV2a & PCV2b & PCV2d & PCV2a/2b & PCV2a/2d & PCV2b/2d \\
\hline Maximum & 100 & 97.9 & 99.8 & 100 & 99.8 & 100 \\
Minimum & 93.4 & 94.6 & 97.9 & 97.7 & 94.5 & 90 \\
\hline
\end{tabular}

Table 3. Results of the detection of recombination events among genomes of 48 PCV2 isolates and 25 other sequences obtained from GenBank using RDP 4.0 molecular recombination software

\begin{tabular}{|c|c|c|c|c|c|}
\hline No. & Recombinant/genotype & Major parent/genotype & Minor parent/genotype & Detection algorithm/P valve & $\begin{array}{l}\text { Breakpoint positions } \\
\text { beginning/ending }\end{array}$ \\
\hline \multirow[t]{7}{*}{1} & KY126314-SHX16 & KY126317-HBXT1 & Unknown (JX519293-PCV2d) & $\mathrm{RDP} / 2.26 \times 10^{-4}$ & $864 / 1763$ \\
\hline & & & & 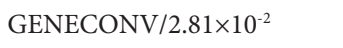 & \\
\hline & & & & Bootscan $/ 8.15 \times 10^{-4}$ & \\
\hline & & & & Maxchi $/ 2.25 \times 10^{-7}$ & \\
\hline & & & & Chimaera/NS & \\
\hline & & & & SiSscan $/ 5.54 \times 10^{-11}$ & \\
\hline & & & & 3 Seq $/ 8.12 \times 10^{-7}$ & \\
\hline \multirow[t]{7}{*}{2} & KY126317-HBXT1 & KX981604-SD-LY & AY713470-PCV2d & $\mathrm{RDP} / 3.24 \times 10^{-3}$ & $1772 / 906$ \\
\hline & & & & GENECONV $/ 4.73 \times 10^{-2}$ & \\
\hline & & & & Bootscan $/ 2.53 \times 10^{-2}$ & \\
\hline & & & & Maxchi $/ 3.35 \times 10^{-7}$ & \\
\hline & & & & Chimaera/5.75 $\times 10^{-3}$ & \\
\hline & & & & SiSscan/NS & \\
\hline & & & & 3 Seq $/ 8.61 \times 10^{-6}$ & \\
\hline \multirow[t]{7}{*}{3} & JX512856-PCV2d & & JX519293-PCV2d & $\mathrm{RDP} / \mathrm{NS}$ & $1152 / 1544$ \\
\hline & & & & GENECONV/NS & \\
\hline & & & & Bootscan/NS & \\
\hline & & & & 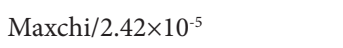 & \\
\hline & & & & Chimaera/NS & \\
\hline & & & & SiSscan $/ 1.41 \times 10^{-5}$ & \\
\hline & & & & 3 Seq $/ 3.93 \times 10^{-2}$ & \\
\hline \multirow[t]{7}{*}{4} & FJ644927-PCV2d & JX535296-PCV2d & KX981602-SD-HJ & $\mathrm{RDP} / 3.79 \times 10^{-5}$ & $1440 / 1643$ \\
\hline & & & & GENECONV $/ 2.35 \times 10^{-4}$ & \\
\hline & & & & Bootscan $/ 3.19 \times 10^{-5}$ & \\
\hline & & & & Maxchi $/ 1.14 \times 10^{-2}$ & \\
\hline & & & & Chimaera/2.75 $\times 10^{-2}$ & \\
\hline & & & & SiSscan $/ 3.88 \times 10^{-4}$ & \\
\hline & & & & 3 Seq $/ 5.55 \times 10^{-5}$ & \\
\hline \multirow[t]{7}{*}{5} & AY754022-PCV2a & JX519293-PCV2d & Unknown (EU148505-PCV2c) & $\mathrm{RDP} / \mathrm{NS}$ & $1266 / 1544$ \\
\hline & & & & GENECONV/NS & \\
\hline & & & & Bootscan/NS & \\
\hline & & & & Maxchi/2.73 $\times 10^{-4}$ & \\
\hline & & & & Chimaera/NS & \\
\hline & & & & SiSscan $/ 4.04 \times 10^{-5}$ & \\
\hline & & & & 3 Seq $/ 4.09 \times 10^{-3}$ & \\
\hline \multirow[t]{7}{*}{6} & JX519293-PCV2d & KX981604-SD-LY & Unknown (AF055394-PCV2b) & RDP/NS & $292 / 864$ \\
\hline & & & & GENECONV/NS & \\
\hline & & & & Bootscan/NS & \\
\hline & & & & Maxchi/2.75 $\times 10^{-4}$ & \\
\hline & & & & Chimaera/NS & \\
\hline & & & & SiSscan $/ 1.6 \times 10^{-7}$ & \\
\hline & & & & $3 \mathrm{Seq} / \mathrm{NS}$ & \\
\hline
\end{tabular}


Table 4. Variable amino acid residues in the capsid protein amongst 48 PCV2 isolates in China from 2016 to 2018

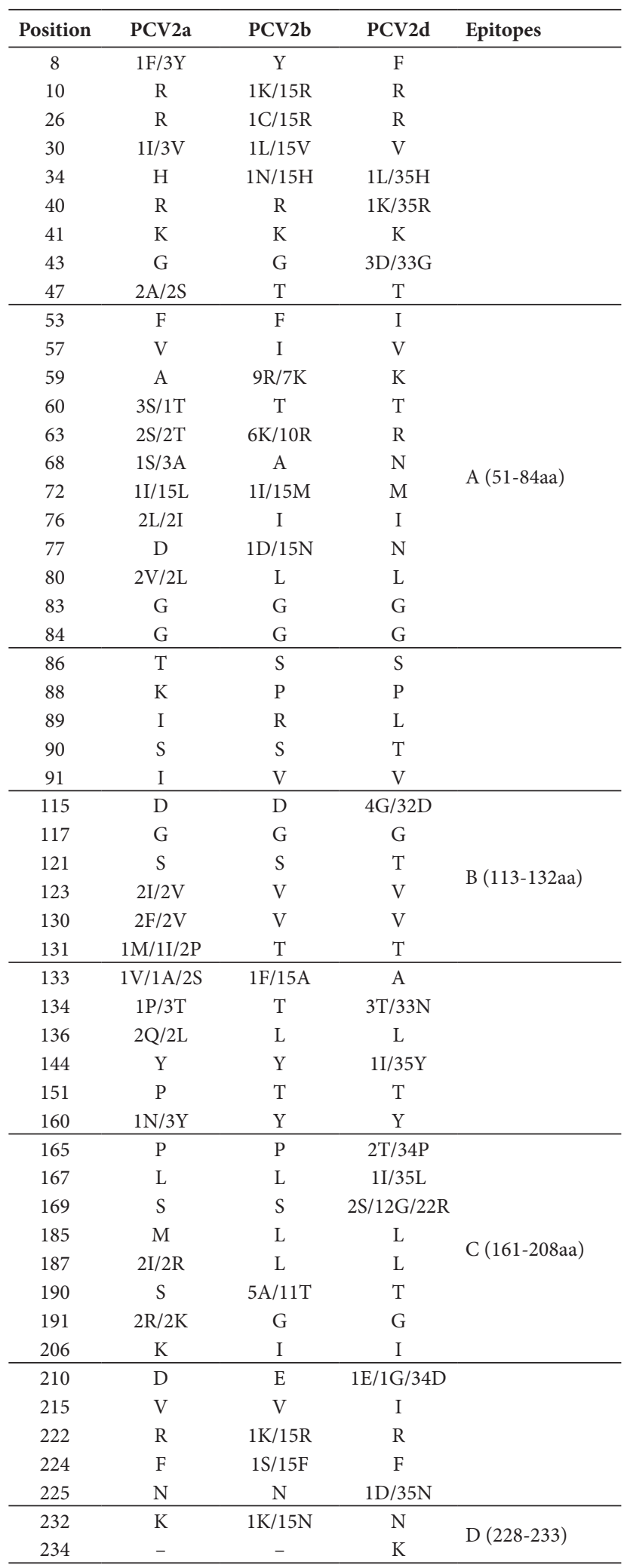

$(-)=$ indicates that amino acid residues at this position are deleted.

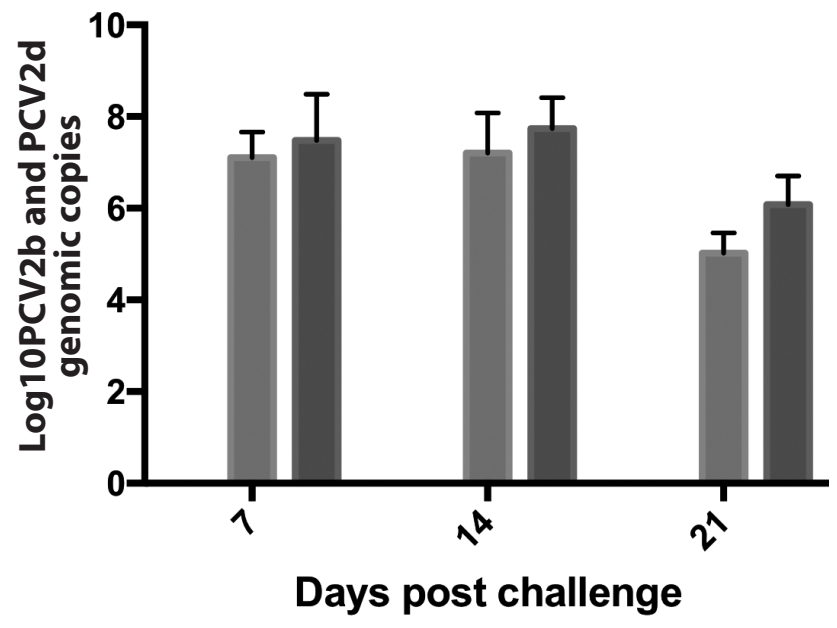

Negative group

PCV2b strain HeB-1 group

- PCV2d strain LN-3 group

Fig. 3

PCV2 DNA in sera after challenge of pigs with PCV2b or PCV2d PCV2b strain HeB-1 or PCV2d strain LN-3 genomic copies in serum were measured at days 7, 14, and 21 after virus challenge and were expressed as the mean $\log _{10}$ quantities of 5 pigs per group. No DNA was detected prior to viral challenge. There were no significant differences between pigs challenged with PCV2b strain HeB-1 or PCV2d strain LN-3. ( $p>0.05)$.

sitions 51-84, 113-136, 166-208, and 228-234, defined as epitopes A-D, respectively (Trible et al., 2011). In this study, 12 variable amino acid residues were detected in the epitope $A$, six in the epitope $B$, eight in the epitope $C$, and two in the epitope D. Furthermore, 14, 2, and 7 amino acid residues were unique to the genotype PCV2a $\left({ }^{47} \mathrm{~A} / \mathrm{S},{ }^{59} \mathrm{~A},{ }^{63} \mathrm{~S} / \mathrm{T},{ }^{86} \mathrm{~T}\right.$, $\left.{ }^{88} \mathrm{~K},{ }^{89} \mathrm{I},{ }^{91} \mathrm{I},{ }^{131} \mathrm{M} / \mathrm{I} / \mathrm{P},{ }^{151} \mathrm{P},{ }^{185} \mathrm{M},{ }^{187} \mathrm{I} / \mathrm{R},{ }^{190} \mathrm{~S},{ }^{191} \mathrm{~K} / \mathrm{R},{ }^{206} \mathrm{~K}\right)$, PCV $2 \mathrm{~b}$ $\left({ }^{57} \mathrm{I},{ }^{89} \mathrm{R}\right)$, and PCV $2 \mathrm{~d}\left({ }^{53} \mathrm{I},{ }^{68} \mathrm{~N},{ }^{89} \mathrm{~L},{ }^{90} \mathrm{~T},{ }^{121} \mathrm{~T},{ }^{215} \mathrm{I},{ }^{234} \mathrm{~K}\right)$, respectively. This suggests a higher amino acid residue diversity at important positions $\left({ }^{43} \mathrm{D} / \mathrm{G},{ }^{115} \mathrm{D} / \mathrm{G},{ }^{134} \mathrm{~N} / \mathrm{T},{ }^{165} \mathrm{P} / \mathrm{T},{ }^{169} \mathrm{G} / \mathrm{R} / \mathrm{S}\right.$, and $\left.{ }^{210} \mathrm{E} / \mathrm{G} / \mathrm{D}\right)$ in the predominant genotype $P C V 2 d$.

Pathogenicity study of the PCV2b strain $\mathrm{HeB}-1$ and PCV2d strain LN-3

To further assess the replication of PCV2b and PCV2d in the sera of infected pigs, we selected HeB-1(PCV2b) and LN-3(PCV2d) to challenge four-week-old piglets. None of the pigs developed clinical signs, and no difference in weight gain was observed between treatment groups. The viral titers of the PCV2d strain LN-3 in sera of pigs were higher than those of the PCV2b strain HeB-1 at 14 and 21 days postchallenge, however the differences were not statistically significant ( $p>0.05$ ) (Fig. 3). There were no obvious differ- 

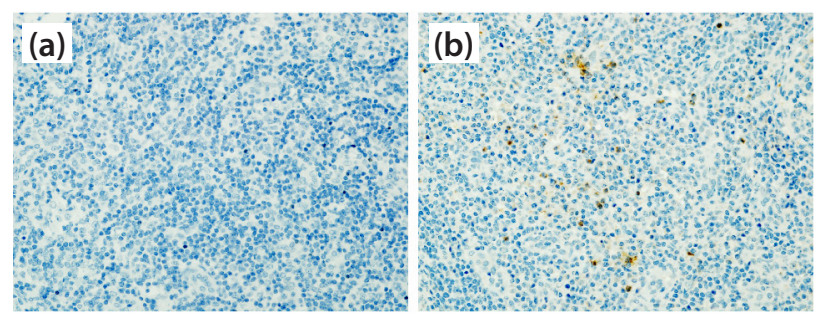

Fig. 4

Immunohistochemical detection of PCV2 antigen in the lymph nodes of experimental pigs

(a) PCV2b antigen was detected in the inguinal lymph nodes from the control group. (b) PCV2b antigen was detected in the inguinal lymph nodes from pigs challenged with PCV2b HeB-1 strain. (c) PCV2d antigen was detected in the inguinal lymph nodes from pigs challenged with PCV2d LN-3 strain. There were no significant differences between pigs challenged with PCV2b strain HeB-1 or PCV2d strain LN-3.

ences between the amount of PCV2 antigen in lymphoid tissues between the strains LN-3 and in the HeB-1 (Fig. 4).

\section{Discussion}

PCV2 is considered to be an essential agent for the pathogenesis of PCVAD, which causes huge economic losses for swine industry worldwide and therefore, a systemic epidemiological surveillance for this disease is essential. PCV2 strains were subdivided into four main genotypes: PCV2a, PCV2b, PCV2c, and PCV2d. A shift from PCV2a to PCV2b had occurred between 2009 and 2010 in China. Currently, PCV2d genotype has become prevalent in China since 2010 (Guo et al., 2010; Cai et al., 2012). The shift of PCV2 genotype may be affected by the immune stress of vaccination. A mass of data was used to analyze the changes of PCV2a and PCV2b genotypes in immune vaccines (based on PCV2a) and non-immune vaccines. The results showed that PCV2a prevalence was reduced from $24.2 \%$ (vaccinated herds) to $3.6 \%$ (non-vaccinated herds), at the same time, PCV2b prevalence increased from $75.8 \%$ (non-vaccinated herds) to 96.4\% (vaccinated herds) (Reiner et al., 2015). These findings were also supported by Shen et al. (2012). The main objective of this work was to evaluate the genotypes of PCV2 strains circulating in the PCV2-affected pigs and healthy pigs in China. The results showed that $64.6 \%$ (31 of 48 ) of the PCV2 strains isolated in China from 2016 to 2018 belong to the PCV2d genotype, indicating dominance of PCV2d among the three genotypes. In addition, $54.5 \%$ isolates belonged to PCV2d in 2016, 65.2\% in 2017, and 71.4\% in 2018. PCV2d showed an increasing tendency and became the dominant genotype in China in 2018. The reason for this situation is probably that China's commercial PCV2 vaccine was developed based on PCV2a and PCV2b. Under the strong vaccine immune pressure, clinical PCV2 genotypes rapidly evolved and shifted to PCV2d genotype.
Recombination is a process with which PCV2 gains genetic diversity and therefore, in combination with point mutations, it plays a crucial role during PCV2 evolution. Recombination is common phenomenon among PCV2 strains, which had been confirmed in several countries (Huang et al., 2013; Reiner et al., 2015). There were no recombinants among the 48 PCV2 isolates in China from 2016 to 2018; however, six recombinants were found in the 25 other sequences obtained from GenBank. It can be concluded that recombination was not the main variation mode of PCV2 in China in recently years.

Alterations of the capsid of PCV2 may affect viral life cycle, antigenicity of capsid proteins, or neutralizing antibody recognition. Epitopes on capsid of PCV were analyzed and divided into four general antibody recognition regions, named A (51 to 84), B (113 to 132), C (161 to 208), and D (228 to 233), respectively (Trible et al., 2011). In the present study, several amino acid mutations occurred in these regions within the 48 isolates, especially at several epitope-associated positions. PCV2d was the major genotype and acquired a change from phenylalanine to isoleucine at position 53 and from serine to threonine at position 121 in these regions. Residues at positions 59-60, 169, and 190-191 located at three conformational epitopes were recognized by neutralizing antibodies (Khayat et al., 2011). For the isolates investigated in this study, mutation occurred at positions 59 and 190 in PCV2b strains, at positions 60 and 191 in PCV2a strains, and at position 169 in PCV2d strains. Our results showed that loop CT formed by nine amino acids from position 225 to 233 could influence the neutralizing antibody binding to the conformational epitope (Khayat et al., 2011). The isolate JX-2 (GenBank accession numbers MH920582) changed from asparagine to lysine at position 232 at the loop CT of the Cap. Furthermore, a PCV2d mutant with an additional lysine at the end of the Loop CT could change the surface patterns of the capsid compared to traditional PCV2b isolates (Zhan et al., 2016). It has been reported that PCV2d with an elongation of lysine showed stronger virulence than PCV2a 
and PCV2b isolates (Guo et al., 2012). All PCV2d strains that we isolated had lysine at position 234. Continuous mutations at these critical positions may help PCV to escape the host immune system and vaccination, thus promoting virus propagation under field conditions.

The selection impact of vaccination on the PCV2 can probably cause the shift in the genotype. Therefore, we speculate that selection impact of PCV2a vaccination causes the prevalence of PCV2d. Currently, commercial vaccines are mainly based on PCV2a and PCV2b, which may be the main reason for the increased prevalence of PCV2d. There may be an unknown evolutionary mechanism of PCV2 to escape vaccine immunization. Although our results showed that there were no obvious differences between the virulence of PCV2d strain LN-3 and the PCV2b strain HeB-1, the changing landscape of PCV2 diversity should be monitored via continual epidemiology surveillance and support should be provided for the development of vaccines. The development of appropriate strategies is required for successful disease control.

\section{References}

Afolabi KO, Iweriebor BC, Obi LC, Okoh AI (2017): Molecular detection of Porcine circovirus type 2 in swine herds of Eastern Cape Province South Africa. Bmc Microbiol. 17, 212. https://doi.org/10.1186/s12866-017-1121-4

Cai L, Ni J, Xia Y, Zi Z, Ning K, Qiu P, Li X, Wang B, Liu Q, Hu D, Yu X, Zhou Z, Zhai X, Han X, Tian K (2012): Identification of an emerging recombinant cluster in porcine circovirus type 2. Virus Res. 165, 95-102. https://doi.org/10.1016/j. virusres.2012.01.008

Cheung AK (2012): Porcine circovirus: transcription and DNA replication. Virus Res. 164, 46-53. https://doi.org/10.1016/j. virusres.2011.10.012

Davies B, Wang X, Dvorak CM, Marthaler D, Murtaugh MP (2016): Diagnostic phylogenetics reveals a new Porcine circovirus 2 cluster. Virus Res. 217, 32-37. https://doi.org/10.1016/j. virusres.2016.02.010

Firth C, Charleston MA, Duffy S, Shapiro B, Holmes EC (2009): Insights into the evolutionary history of an emerging livestock pathogen: porcine circovirus 2. J. Virol. 83, 12813-12821. https://doi.org/10.1128/JVI.01719-09

Franzo G, Cortey M, Segales J, Hughes J, Drigo M (2016): Phylodynamic analysis of porcine circovirus type 2 reveals global waves of emerging genotypes and the circulation of recombinant forms. Mol. Phylogenet. Evol. 100, 269-280. https://doi.org/10.1016/j.ympev.2016.04.028

Guo L, Fu Y, Wang Y, Lu Y, Wei Y, Tang Q, Fan P, Liu J, Zhang L, Zhang F, Huang L, Liu D, Li S, Wu H, Liu C (2012): A porcine circovirus type 2 (PCV2) mutant with 234 amino acids in capsid protein showed more virulence in vivo, compared with classical PCV2a/b strain. PloS One 7, e41463. https://doi.org/10.1371/journal.pone.0041463
Guo LJ, Lu YH, Wei YW, Huang LP, Liu CM (2010): Porcine circovirus type 2 (PCV2): genetic variation and newly emerging genotypes in China. Virol. J. 7, 273. https://doi. org/10.1186/1743-422X-7-273

Huang Y, Shao M, Xu X, Zhang X, Du Q, Zhao X, Zhang W, Lyu Y, Tong D (2013): Evidence for different patterns of natural inter-genotype recombination between two PCV2 parental strains in the field. Virus Res. 175, 78-86. https://doi. org/10.1016/i.virusres.2013.03.014

Khayat R, Brunn N, Speir JA, Hardham JM, Ankenbauer RG, Schneemann A, Johnson JE (2011): The 2.3-angstrom structure of porcine circovirus 2. J. Virol. 85, 7856-7862. https://doi.org/10.1128/JVI.00737-11

Kumar S, Stecher G, Tamura K (2016): MEGA7: Molecular Evolutionary Genetics Analysis Version 7.0 for Bigger Datasets. Mol. Biol. Evol. 33, 1870-1874. https://doi.org/10.1093/ molbev/msw054

Kwon T, Lee DU, Yoo SJ, Je SH, Shin JY, Lyoo YS (2017): Genotypic diversity of porcine circovirus type 2 (PCV2) and genotype shift to PCV2d in Korean pig population. Virus Res. 228, 24-29. https://doi.org/10.1016/j.virusres.2016.11.015

Li J, Shi JL, Wu XY, Fu F, Yu J, Yuan XY, Peng Z, Cong XY, Xu SJ, Sun WB, Cheng KH, Du YJ, Wu JQ, Wang JB, Huang BH (2015): Improvement of the Immunogenicity of Porcine Circovirus Type 2 DNA Vaccine by Recombinant ORF2 Gene and CpG Motifs. Viral Immunol. 28, 290-296. https://doi.org/10.1089/vim.2014.0121

Lv Q, Guo K, Xu H, Wang T, Zhang Y (2015): Correction: Identification of Putative ORF5 Protein of Porcine Circovirus Type 2 and Functional Analysis of GFP-Fused ORF5 Protein. PloS One 10, e127859. https://doi.org/10.1371/ journal.pone. 0134203

Martin DP, Murrell B, Golden M, Khoosal A, Muhire B (2015): RDP4: Detection and analysis of recombination patterns in virus genomes. Virus Evol. 1, v3. https://doi. org/10.1093/ve/vev003

Nawagitgul P, Morozov I, Bolin SR, Harms PA, Sorden SD, Paul PS (2000): Open reading frame 2 of porcine circovirus type 2 encodes a major capsid protein. J. Gen. Virol. 81, 22812287. https://doi.org/10.1099/0022-1317-81-9-2281

Olvera A, Cortey M, Segales J (2007): Molecular evolution of porcine circovirus type 2 genomes: phylogeny and clonality. Virology 357, 175-185. https://doi.org/10.1016/j. virol.2006.07.047

Opriessnig T, Meng XJ, Halbur PG (2007): Porcine circovirus type 2 associated disease: update on current terminology, clinical manifestations, pathogenesis, diagnosis, and intervention strategies. J. Vet. Diagn. Invest. 19, 591-615. https://doi. org/10.1177/104063870701900601

Opriessnig T, O'Neill K, Gerber PF, de Castro AM, Gimenez-Lirola LG, Beach NM, Zhou L, Meng XJ, Wang C, Halbur PG (2013): A PCV2 vaccine based on genotype $2 b$ is more effective than a 2a-based vaccine to protect against PCV2b or combined PCV2a/2b viremia in pigs with concurrent PCV2, PRRSV and PPV infection. Vaccine 31, 487-494. https://doi.org/10.1016/j.vaccine.2012.11.030 
Ramos N, Mirazo S, Castro G, Arbiza J (2013): Molecular analysis of Porcine Circovirus Type 2 strains from Uruguay: evidence for natural occurring recombination. Infect. Genet. Evol. 19, 23-31. https://doi.org/10.1016/j.meegid.2013.06.017

Reiner G, Hofmeister R, Willems H (2015): Genetic variability of porcine circovirus 2 (PCV2) field isolates from vaccinated and non-vaccinated pig herds in Germany. Vet. Microbiol. 180, 41-48. https://doi.org/10.1016/j.vetmic.2015.07.026

Rose N, Andraud M, Bigault L, Jestin A, Grasland B (2016): A commercial PCV2a-based vaccine significantly reduces PCV2b transmission in experimental conditions. Vaccine 34, 3738-3745. https://doi.org/10.1016/j.vaccine.2016.06.005

Segales J, Kekarainen T, Cortey M (2013): The natural history of porcine circovirus type 2: from an inoffensive virus to a devastating swine disease? Vet. Microbiol. 165, 13-20. https://doi.org/10.1016/j.vetmic.2012.12.033
Shen HG, Halbur PG, Opriessnig T (2012): Prevalence and phylogenetic analysis of the current porcine circovirus 2 genotypes after implementation of widespread vaccination programmes in the USA. J. Gen. Virol. 93, 1345-1355. https://doi.org/10.1099/vir.0.039552-0

Tischer I, Gelderblom H, Vettermann W, Koch MA (1982): A very small porcine virus with circular single-stranded DNA. Nature 295, 64-66. https://doi.org/10.1038/295064a0

Trible BR, Kerrigan M, Crossland N, Potter M, Faaberg K, Hesse R, Rowland RR (2011): Antibody recognition of porcine circovirus type 2 capsid protein epitopes after vaccination, infection, and disease. Clin. Vaccine Immunol. 18, 749-757. https://doi.org/10.1128/CVI.00418-10

Zhan Y, Wang N, Zhu Z, Wang Z, Wang A, Deng Z, Yang Y (2016): In silico analyses of antigenicity and surface structure variation of an emerging porcine circovirus genotype 2b mutant, prevalent in southern China from 2013 to 2015. J. Gen. Virol. 97, 922-933. https://doi.org/10.1099/ jgv. 0.000398 